\title{
ADESÃO À HIGIENE DAS MÃOS: INTERVENÇÃO E AVALIAÇÃO
}

\author{
Karen Patricia Pena Trannin¹, Cássia Regina Vancini Campanharo², Maria Carolina Barbosa Teixeira Lopes ${ }^{3}$, \\ Meiry Fernanda Pinto Okuno ${ }^{4}$, Ruth Ester Assayag Batista ${ }^{5}$
}

\begin{abstract}
RESUMO: Trata-se de pesquisa quantitativa, com os objetivos de observar a adesão à higiene das mãos por profissionais de saúde de um Serviço de Emergência de Hospital Universitário, no estado de São Paulo, e verificar se houve modificação na adesão após a realização de intervenção educativa, entre julho de 2012 e dezembro de 2013. Foram observadas e registradas 5061 oportunidades de higiene das mãos em 120 horas de observação. O maior número de oportunidades foi de profissionais de enfermagem (70,05\%), por ser a maior força de trabalho e tender predominantemente à assistência; seguiram-se a equipe médica $(17,82 \%)$ e fisioterapeutas $(12,13 \%)$. Observou-se adesão de $28,6 \%$ para $38,9 \%$ após as ações educativas. Na fase pós-intervenção, todos os profissionais apresentaram maior adesão à higiene das mãos quando comparado ao período pré-intervenção e a adesão foi significativamente maior após a realização de procedimentos assépticos. Conclui-se que a higienização das mãos esteve aquém do esperado e que estratégias educativas favoreceram a adesão.
\end{abstract}

DESCRITORES: Higiene das mãos; Infecção hospitalar; Pessoal de saúde.

\section{ADHERENCE TO HAND HYGIENE: INTERVENTION AND ASSESSMENT}

ABSTRACT: Quantitative study aimed to observe adherence to hand hygiene by health professionals of an Emergency Care Service of a Teaching Hospital, in the state of São Paulo, and assess whether an educational intervention conducted between July 2012 and December 2013 had impact on this practice. During 120 hours of observation, 5,061 opportunities of hand hygiene were observed and recorded. The greatest number of opportunities was observed among nursing professionals (70.05\%), because they are most numerous health professionals and usually perform care functions; these were followed by the medical team (17.82\%) and physiotherapists $(12.13 \%)$. After the educational activities adherence increased from $28.6 \%$ to $38.9 \%$. In the post-intervention moment, all the professionals showed higher adherence to hand hygiene compared to the pre-intervention moment, and adherence was significantly higher after the use of aseptic procedures. It is concluded that hand hygiene compliance was less than expected and educational strategies favored adhesion.

DESCRIPTORS: Hand hygiene; Hospital infection; Health personnel.

\section{ADHESIÓN A LAHIGIENE DE LAS MANOS: INTERVENCIÓN Y EVALUACIÓN}

RESUMEN: Esta es una investigación cuantitativa cuyas finalidades fueron observar la adhesióna la higiene de las manos por profesionales de salud de un Servicio de Emergencia de Hospital Universitario, en estado de São Paulo, así como verificar si hubo cambio en la adhesión después de la realización de intervención educativa, entre julio de 2012 y diciembre de 2013. Fueron observadas y registradas 5061 oportunidades de higiene de las manos en 120 horas de observación. El mayor número de oportunidades fue de profesionales de enfermería $(70,05 \%)$, en razón de esta ser la mayor fuerza de trabajo y presentar predominancia de asistencia; siguiéndose el equipo médico (17,82\%) y los fisioterapeutas (12,13\%). Se observó adhesión de 28,6\% para 38,9\% después de acciones educativas. En la etapa depos intervención, todos los profesionales presentaron más adhesión a la higiene de las manos cuando comparado al periodo de preintervención, además de la adhesión ser mucho mayor después de la realización de procedimientos asépticos. Se concluye que la higienización de las manos estubo abajo de lo que se espera y que estrategias educativas favorecen la adhesión.

DESCRIPTORES: Higiene de las manos; Infección hospitalar; Personal de salud.

${ }^{1}$ Enfermeira. Especialista em Urgência e Emergência. Escola Paulista de Enfermagem da Universidade Federal de São Paulo. São Paulo, SP, Brasil.

${ }^{2}$ Enfermeira. Doutora em Ciências. Escola Paulista de Enfermagem da Universidade Federal de São Paulo. São Paulo, SP, Brasil. ${ }^{3}$ Enfermeira. Mestre em Ciências. Escola Paulista de Enfermagem da Universidade Federal de São Paulo. São Paulo, SP, Brasil.

${ }^{4}$ Enfermeira. Doutora em Ciências. Escola Paulista de Enfermagem da Universidade Federal de São Paulo. São Paulo, SP, Brasil. ${ }^{5}$ Enfermeira. Doutora em Ciências. Docente da Universidade Federal de São Paulo. São Paulo, SP, Brasil 


\section{- INTRODUÇÃO}

A Infecção Relacionada à Assistência de Saúde (IRAS) constitui mundialmente um dos maiores problemas de segurança do paciente a ser enfrentado, sendo que a higienização das mãos (HM) é comprovadamente a principal medida e um dos pilares mais relevantes para a prevenção de infecções dessa natureza ${ }^{(1)}$.

As mãos estão envolvidas em todo o processo de atendimento tornando-se o principal veículo de transmissão de micro-organismos. Com o objetivo de tornar este contato seguro, a HM é recomendada antes e após o contato com o paciente e seu ambiente, constituindo uma norma básica do ambiente hospitalar e apontada como fundamental à prevenção de infecções nasocomiais ${ }^{(2)}$.

A adesão integral a essa prática vem sendo apontada como de difícil implantação principalmente no serviço de emergência dos hospitais, onde muitas barreiras à higiene adequada das mãos têm sido relatadas por profissionais de saúde, como por exemplo: a falta de tempo, processo de trabalho que requer agilidade e urgência, grande demanda e atendimento simultâneo a vários pacientes ${ }^{(1)}$.

A medida da adesão à HM nos serviços de saúde é uma tarefa extremamente desafiadora, pois não existe consenso sobre um padrão metodológico a ser adotado, sendo realizada por vários métodos, incluindo a observação direta. A medida ideal de aderência seria o número de vezes que o profissional da saúde realmente higienizou suas mãos, dividido pelo número de oportunidades que ele teve para realizá-la. A Organização Mundial da Saúde identificou essas oportunidades como os cinco momentos: antes do contato com o paciente; antes de um procedimento asséptico, depois de um risco de exposição a secreções corporais; após o contato com o paciente e após o contato com o ambiente e objetos do paciente no local onde ele se encontrava ${ }^{(3)}$.

A melhor taxa de adesão à HM exige a integração do controle de infecção com a cultura de segurança da organização, que se refere a um ambiente de trabalho onde um compromisso com a segurança compartilhado por parte da administração e dos trabalhadores é compreendido e seguido ${ }^{(4)}$.

Algumas áreas em potencial devem ser trabalhadas para elevar a adesão à HM entre os profissionais da saúde, como educação do profissional, direcionando as ações para como, quando e por que realizá-la, enfatizando os padrões de comportamento da sociedade e motivação, apoiando modelos de comportamento entre os colegas de profissão ${ }^{(3)}$.

Assim sendo, a HM deve ser prioridade da instituição, devendo ter suporte contínuo da administração do serviço de saúde. As infecções nos serviços de saúde ameaçam tanto os pacientes quanto os profissionais, podendo implicar em processos e indenizações judiciais, nos casos comprovados de negligência durante a assistência prestada(5).

O controle de infecções nos serviços, incluindo as práticas da HM, além de atender as exigências legais e éticas, concorre também para melhoria da qualidade no atendimento e assistência ao paciente. Os benefícios destas práticas são inquestionáveis, desde a redução da morbidade e mortalidade dos pacientes, até a diminuição de custos associados ao tratamento dos quadros infecciosos gerados ${ }^{(5)}$.

A perspectiva de novos estudos científicos e descobertas objetivando a realidade de cada unidade de saúde, juntamente com equipes multiprofissionais, podem levar a mudanças no perfil atual de adesão profissional à HM e suas implicações na transmissão de doenças ${ }^{(6)}$.

Especificamente no Serviço de Emergência, as infecções são favorecidas pela necessidade de realização rápida de procedimentos invasivos, pela gravidade dos pacientes nele atendidos, pela grande demanda, somadas ao comportamento dos profissionais. Sendo assim, medidas simples como a HM podem reduzir a disseminação de microrganismos, contribuindo desta forma para a segurança dos pacientes. A colonização das mãos dos profissionais, que pode chegar a $39 \%$, pode aumentar assim o risco de infecção nesses pacientes ${ }^{(7)}$.

Diante do exposto, este estudo teve por objetivos observar a adesão à HM pelos profissionais da saúde de um Serviço de Emergência de um Hospital Universitário de São Paulo e avaliar se houve modificação após a realização de intervenção educativa. 
Trata-se de uma pesquisa quase-experimental, com abordagem quantitativa, de objetivo explicativa, realizada no Serviço de Emergência de um Hospital Universitário de São Paulo, de julho de 2012 a dezembro de 2013.

O estudo foi aprovado pelo Comitê de Ética e Pesquisa da UNIFESP em 2011 (CEP- 2067/11) e, por não apresentar riscos, desconfortos e garantir o anonimato dos participantes, não foi necessário o uso do Termo de Consentimento Livre e Esclarecido.

A amostra foi composta pelas oportunidades de HM, representadas pelos momentos durante o processo de cuidado, em que a HM foi realizada. Uma oportunidade existe sempre que pelo menos uma das indicações de lavagem das mãos é presente e observada, entretanto, pode ocorrer mais de uma indicação para uma oportunidade única ${ }^{(8)}$.

A coleta de dados foi realizada por meio da observação direta da prática da HM das equipes médicas, de enfermagem e da fisioterapia. Esta estratégia tem sido considerada, pela Organização Mundial da Saúde, o "padrão ouro" para a monitorização do comportamento. Esta técnica consiste na inserção de um observador no ambiente de trabalho, para que este avalie se o profissional de saúde está realizando a prática de acordo com o recomendado, na forma e momento adequados. Entretanto, um fator negativo referente a essa metodologia trata-se da mudança de atitude dos profissionais pelo fato de estarem sendo observados ${ }^{(1)}$.

A observação quantitativa, nesta pesquisa, observou a $\mathrm{HM}$ apenas de acordo com o número de oportunidades e a taxa de aderência à medida, excluindo-se, portanto, aspectos relacionados à qualidade de limpeza das mãos, tempo dispensado para tal, uso de produtos diversos, de luvas ou de adornos.

A amostra do estudo foi por conveniência e a coleta de dados não identificava os profissionais. A seleção ocorreu pela maior força de trabalho no setor de saúde, equipe de enfermagem (55\%), compondo o restante da amostra os médicos (40\%) e fisioterapeutas (5\%).

A coleta de dados ocorreu em dois momentos denominados pré e pós-intervenção, sendo o primeiro constituído de 60 horas de observação totalizando 2304 oportunidades. Todos os profissionais do Serviço de Emergência, que não estavam de licença ou férias, participaram da intervenção educativa.

As atividades educacionais tiveram duração de uma semana com quatro estratégias que incluíram a apresentação dos dados coletados sobre as taxas de HM aos profissionais de saúde; um filme sobre HM; e foram distribuído cartazes do Ministério da Saúde pelo setor, em locais estratégicos. A cada participante foi fornecido um broche colorido para lembrete e estimulação da ação com a imagem e a mensagem "Lave as suas mãos", além de um frasco de álcool gel para incentivo à adesão.

Após um mês, iniciou-se o segundo momento com uma nova coleta de dados no pós-intervenção, com o mesmo total de 60 horas de observação, porém com uma amostra de 2757 oportunidades.

Nos dois momentos a observação foi realizada três dias por semana, por cerca de três horas em cada período do dia (matutino, vespertino e noturno), totalizando 120 horas de observação e alcançando 5.061 oportunidades $^{(9)}$.

O instrumento de coleta elaborado pelos pesquisadores, com base no Guideline for Hand Hygiene in Health-Care Settings, foi composto pelas seguintes variáveis, que correspondiam aos procedimentos com oportunidade de HM: preparo de medicação via oral e endovenosa, administração de medicação, sondagem vesical, sinais vitais, administração de dieta, punção venosa, curativo, sondagem gástrica/ enteral, aspiração, banho, troca de fralda, exame físico, antes ou após o procedimento. O formulário era preenchido pelo pesquisador por meio de observação direta e foi garantido o anonimato sobre a HM, ou não, pelos profissionais do serviço. Foi realizado um teste-piloto com o formulário durante uma semana ${ }^{(10)}$.

Os dados foram armazenados em planilha do programa Excel versão Microsoft Office Excel 2010. Para a análise descritiva dos dados, calculou-se frequência e percentual. As variáveis categóricas foram 
comparadas com as variáveis de interesse por meio do teste qui-quadrado e Análise de Variância (ANOVA) com dois fatores para a variável momento em relação à intervenção e grupos. O nível de significância adotado foi $\mathrm{p}<0,05$.

\section{RESULTADOS}

No período pré e pós-intervenção educacional, este estudo totalizou 120 horas de observação, nas quais foram incluídas 5061 oportunidades para a realização da HM pelos profissionais. O maior número de oportunidades observadas foi dos profissionais da equipe de enfermagem (3545; 70,05\%), seguida da equipe médica e dos fisioterapeutas (902; 17,82\% e $614 ; 12,13 \%)$.

Os procedimentos com maior número de oportunidades foram: exame físico (804; 15,89\%), verificação dos sinais vitais $(672 ; 13,28 \%)$, punção venosa $(572 ; 11,30 \%)$, preparo de medicação endovenosa (533;10,53\%), preparo de medicação via oral $(516 ; 10,20 \%)$, administração de medicação (466; 9,21\%), administração de dieta $(427 ; 8,44 \%)$, troca de fraldas $(270 ; 5,33 \%)$, alguns procedimentos como curativo, banho, oxigenoterapia, sondagem vesical, higiene oral e sondagem gástrica tiveram um percentual menor que $5 \%$ da amostra.

No decorrer de toda pesquisa, no total, os fisioterapeutas apresentaram maior adesão à HM quando comparado outros profissionais, enfermagem e médicos $(p=0,0029)$, conforme apresentado na Tabela 1.

No período pré-intervenção, foram auditadas 2304 (45,52\%) e no pós-intervenção 2757 (54,48\%) oportunidades. Na fase pós-intervenção, todos os profissionais, enfermagem, médicos e fisioterapeutas, apresentaram maior adesão à HM quando comparado ao período pré-intervenção $(p<0,0001)$ (Tabela 2).

O número de oportunidade HM após a realização dos procedimentos foi de 2.572 (50,82\%) e antes dos mesmos foram 2.489 (49,18\%). Houve maior adesão à HM após a realização dos procedimentos $(\mathrm{p}<0,0001)$ (Tabela 3).

Tabela 1 - Total de distribuição do número e da porcentagem da frequência da adesão à higienização das mãos dos grupos profissionais $(\mathrm{n}=5061)$. São Paulo-SP-Brasil, 2013

\begin{tabular}{|c|c|c|c|c|c|}
\hline & \multicolumn{5}{|c|}{ Adesão } \\
\hline & \multicolumn{2}{|c|}{ Sim } & \multicolumn{2}{|c|}{ Não } & \multirow[b]{2}{*}{ p-valor* } \\
\hline & $\mathbf{n}$ & $\%$ & $\mathbf{n}$ & $\%$ & \\
\hline Enfermagem & 1.227 & 34,6 & 2.318 & 65,4 & $p=0,0029$ \\
\hline Médicos & 271 & 30 & 631 & 70 & \\
\hline Fisioterapeutas & 235 & 38,3 & 379 & 61,7 & \\
\hline Total & 1.733 & 34,2 & 3.328 & 65,8 & \\
\hline
\end{tabular}

* Nível de significância de 5\% (p-valor<0,05).

Tabela 2 - Distribuição da frequência e porcentagem da adesão à higienização das mãos antes e após a intervenção educacional ( $\mathrm{n}=5061)$. São Paulo-SP-Brasil, 2013

\begin{tabular}{|c|c|c|c|c|c|}
\hline & \multicolumn{5}{|c|}{ Adesão } \\
\hline & \multicolumn{2}{|c|}{ Sim } & \multicolumn{2}{|c|}{ Não } & $\mathbf{p}$ \\
\hline & $\mathbf{N}$ & $\%$ & $\mathbf{N}$ & $\%$ & \\
\hline Pré - intervenção & 660 & 28,6 & 1.644 & 71,4 & $p<0,0001$ \\
\hline Pós - intervenção & 1.073 & 38,9 & 1.684 & 61,1 & \\
\hline Total & 1.733 & 34,2 & 3.328 & 65,8 & \\
\hline
\end{tabular}


Foram 3.018 (59,63\%) procedimentos não assépticos e 2.043 (40,37\%) assépticos, houve adesão à HM significativamente maior aos procedimentos assépticos $(p=0,0007)$. (Tabela 4$)$

No momento pré-intervenção, a equipe de enfermagem apresentou maior percentual de adesão à HM com variação significativa entre os fisioterapeutas e médicos $(p=0,0010)$. Entretanto, no momento pós-intervenção, foram os fisioterapeutas que tiveram maior adesão à HM ( $p=0,0000)$ (Tabela 5).

Todos os profissionais apresentaram um aumento considerável na adesão à HM após a intervenção.

Tabela 3 - Distribuição da frequência e porcentagem da adesão à higienização das mãos antes e após o procedimento $(\mathrm{n}=5061)$. São Paulo-SP-Brasil, 2013

\begin{tabular}{|c|c|c|c|c|c|}
\hline \multirow{3}{*}{$\begin{array}{l}\text { Momento da higiene das } \\
\text { mãos }\end{array}$} & \multicolumn{5}{|c|}{ Adesão } \\
\hline & \multicolumn{2}{|c|}{ Sim } & \multicolumn{2}{|c|}{ Não } & p \\
\hline & $\mathbf{N}$ & $\%$ & $\mathbf{n}$ & $\%$ & \\
\hline Antes do procedimento & 467 & 18,8 & 2.022 & 81,2 & $P<0,0001$ \\
\hline Depois do procedimento & 1.266 & 49,2 & 1.306 & 50,8 & \\
\hline Total & 1733 & 34,2 & 3.328 & 65,8 & \\
\hline
\end{tabular}

Tabela 4 - Distribuição da frequência e porcentagem a adesão à higienização das mãos em relação aos procedimentos assépticos e não asséptico ( $\mathrm{n}=5061)$. São Paulo-SP-Brasil, 2013

\begin{tabular}{lccccc}
\multirow{2}{*}{ Procedimento } & \multicolumn{6}{c}{ Adesão } \\
\cline { 2 - 7 } & \multicolumn{2}{c}{ Sim } & \multicolumn{3}{c}{ Não } \\
\cline { 2 - 7 } & $\mathbf{N}$ & \% & n & \% & p-valor \\
\hline Não assépticos & 977 & 32,3 & 2041 & 67,6 & 0,001 \\
\hline Assépticos & 756 & 37 & 1287 & 63 & \\
\hline Total & 1733 & 34,2 & 3.328 & 65,76
\end{tabular}

Tabela 5 - Distribuição da frequência e porcentagem a adesão à higienização das mãos no período pré e pósintervenção educacional $(n=5061)$. São Paulo-SP-Brasil, 2013

\begin{tabular}{|c|c|c|c|c|c|c|c|c|c|}
\hline \multirow[t]{4}{*}{ Grupos } & \multicolumn{9}{|c|}{ Adesão à higiene das mãos } \\
\hline & \multicolumn{4}{|c|}{ Pré-intervenção } & \multicolumn{5}{|c|}{ Pós-intervenção } \\
\hline & \multicolumn{2}{|c|}{ Sim } & \multicolumn{2}{|c|}{ Não } & \multicolumn{2}{|c|}{ Sim } & \multicolumn{2}{|c|}{ Não } & \multirow[t]{2}{*}{$\mathbf{p}$} \\
\hline & $\mathbf{N}$ & $\%$ & $\mathbf{N}$ & $\%$ & $\mathbf{n}$ & $\%$ & $\mathbf{N}$ & $\%$ & \\
\hline Enfermagem & 506 & 31,2 & 1117 & 68,8 & 721 & 37,5 & 1201 & 62,5 & 0,001 \\
\hline Médicos & 87 & 21,9 & 311 & 78,1 & 184 & 36,5 & 320 & 63,5 & $<0,0001$ \\
\hline Fisioterapeutas & 67 & 23,7 & 216 & 76,3 & 168 & 80,8 & 163 & 49,2 & $<0,0001$ \\
\hline Total & 660 & 28,6 & 1644 & 71,4 & 1073 & 38,9 & 1684 & 61,1 & \\
\hline
\end{tabular}

\section{DISCUSSÃO}

Monitorar a adesão à HM diminui a transmissão de patógenos e, sobretudo, a incidência de infecções relacionadas ao cuidar em saúde, sendo considerada uma medida simples e importante na redução de mortalidade entre os pacientes ${ }^{(6)}$.

Neste estudo, o maior número de oportunidades observadas para a HM foi a dos profissionais da equipe de enfermagem, seguida da equipe médica e dos fisioterapeutas. Resultado semelhante foi encontrado por outro estudo em que $75,3 \%$ das oportunidades para a HM foram auditadas na equipe de enfermagem, por consistir a maior força de trabalho nos serviços de saúde, devido ao fato de tender predominantemente à assistência ${ }^{(11)}$. 
Os procedimentos com maior número de oportunidades para HM neste estudo foram: exame físico, verificação dos sinais vitais, punção venosa e preparo de medicação endovenosa, respectivamente. Estes resultados diferem de outro estudo realizado no Pronto Socorro de um hospital universitário, em que se observou que os procedimentos mais realizados foram verificação de sinais vitais $(80,8 \%)$, punções venosas $(76,6 \%)$ e oxigenoterapia $(40,4 \%)^{(12)}$.

Nesta pesquisa, os fisioterapeutas apresentaram maior adesão à $\mathrm{HM}$, seguidos pela enfermagem e médicos, divergindo de outra pesquisa em que os enfermeiros aderiram mais do que os técnicos de enfermagem e médicos na fase pré-intervenção; porém, somente na categoria dos técnicos de enfermagem houve aumento significativo na adesão pós-intervenção(13).

Na fase pós-intervenção, os profissionais deste estudo apresentaram adesão à HM significativamente maior quando comparado com o período pré-intervenção. Pesquisa em uma Unidade de Terapia Intensiva no noroeste do Paraná também constatou melhora significativa na taxa de adesão global à HM após uma estratégia de promoção à prática entre técnicos de enfermagem, enfermeiros e médicos ${ }^{(13)}$.

Estudo realizado em 603 procedimentos de um hospital escola da Universidade Federal de São Carlos observou que em 238 a HM foi realizada exclusivamente após os procedimentos ${ }^{(14)}$, reforçando os nossos achados de que a adesão é significativamente maior após a realização de procedimentos e nos procedimentos assépticos.

No período pré-intervenção educacional, as proporções da adesão dos enfermeiros foi superior à dos médicos e fisioterapeutas. Entretanto, no momento pós-intervenção, a categoria de fisioterapeutas foi a que apresentou maior adesão à $\mathrm{HM}$, seguida pela enfermagem e médica. Estudo realizado em uma Unidade de Clínica Médica e Cirúrgica de um hospital em Minas Gerais também evidenciou variação da adesão de acordo com a categoria à qual o profissional de saúde pertencia, tendo os técnicos e auxiliares de enfermagem realizado mais vezes a técnica de lavagem de mãos $(73,9 \%)$, seguidos dos médicos $(13,1 \%)$, fisioterapeutas $(8,7 \%)$ e enfermeiros $(4,3 \%)^{(15)}$.

O procedimento da técnica de HM é a mais simples e importante medida de controle de infecção, entretanto comprova-se através dos dados coletados que os profissionais de saúde não realizam a técnica recomendada. A baixa adesão à $\mathrm{HM}$ não está diretamente associada ao conhecimento teórico, mas à incorporação desse conhecimento na prática diária. É observado que durante campanhas de $\mathrm{HM}$, frequentemente ocorre aumento da adesão, que retorna aos níveis basais geralmente seis meses após a campanha, refletindo um problema não só estrutural, mas também de conscientização e ética dos profissionais ${ }^{(16)}$.

Os serviços de urgência e emergência têm peculiaridades e especificidades que têm sido objeto de estudo em todo mundo, com enfoque especial para questões relativas ao aumento da demanda, evidenciando problemas relacionados à infraestrutura, organização dos serviços, qualidade da assistência, quantidade e especificidade dos atendimentos realizados ${ }^{(17)}$.

A avaliação contínua da conformidade à adesão a HM no serviço hospitalar é extremamente importante e deve ser realizada continuamente para garantir que as intervenções tenham o impacto desejado, além de identificar as áreas e os profissionais que requerem aperfeiçoamento ${ }^{(16)}$.

\section{CONCLUSÃO}

Neste estudo os profissionais de saúde apresentaram maior adesão à HM no momento pósintervenção, após a realização dos procedimentos e nos procedimentos assépticos. Todos os profissionais apresentaram um aumento considerável na adesão à HM após a intervenção.

O estudo apresenta como limitação o fato de não disponibilizar dados sobre a técnica utilizada para HM.

Diante do exposto, ressalta-se a importância de intervenções educacionais em HM nos serviços de saúde, em especial nas unidades de atendimento a urgência e emergência, com o intuito de garantir a qualidade do cuidado na assistência prestada, além de promover a segurança aos pacientes e consequentemente reduzir custos hospitalares. Neste contexto, há necessidade do Enfermeiro 
apropriar-se da sua função educadora, como recurso essencial para promoção e prevenção de infecções relacionada à assistência.

\section{REFERÊNCIAS}

1. di Martino P, Ban KM, Bartoloni A, Fowler KE, Saint S, Mannelli F. Assessing the sustainability of hand hygiene adherence prior to patient contact in the emergency department: A 1-year post intervention evaluation. Am J Infect Control. 2011; 39(1): 14-8.

2. Guedes M, Miranda FMDA, Maziero ECS, Cauduro FLF, Cruz EDA. Adesão dos profissionais de enfermagem à higienização das mãos: uma análise segundo o modelo de crenças em saúde. Cogitare Enferm. 2012; 17(2): 304-9.

3. Steed C, Kelly W, Blackhurst D, Boeker S, Diller T, Alper P, et al. Hospital hand hygiene opportunities: where and when (HOW2)? The HOW2 Benchmark Study. Am J Infect Control. 2011; 39(1): 19-26.

4. Siegel JD, Rhineheart E, Jackson M, Chiarello L. Health Care Infection Control Practices Advisory CommitteeAJIC. Guideline for isolation precautions: preventing transmission of infectious agents in health care settings. Am J Infect Control. 2007; 35(10): 65-164.

5. Borges Primo MG, Ribeiro LCM, Figueiredo LFS, Sirico SCA, de Souza MA. Adesão à prática de higienização das mãos por profissionais de saúde de um hospital universitário. Rev. Eletr. Enf. 2010; 12(2): 266-71.

6. de Oliveira AC, de Paula AO. Monitorização da adesão à higiene das mãos: uma revisão de literatura. Acta Paul. Enferm. 2011; 24(3): 407-13.

7. Koçak Tufan Z, Irmak H, Bulut C, Cesur S, Kinikli S, Demiröz AP. The effectiveness of hand hygiene products on MRSA colonization of health care workers by using CHROMagar MRSA. Mikrobiyol Bul. 2012; 46(2): 236-46.

8. Ministério da Saúde (BR). Agência Nacional de Vigilância Sanitária. Manual para observadores: estratégia multimodal da OMS para a melhoria da higienização das mãos. / Organização Mundial da Saúde. Brasília (DF): Organização Pan-Americana da Saúde; Agência Nacional de Vigilância Sanitária; 2008.

9. Barbosa LR. Correlação entre métodos de mensuração da adesão à higiene das mãos em unidade de terapia intensiva neonatal (tese). São Paulo (SP): Universidade de São Paulo; 2010.

10. Boyce JM, Pittet D. Guideline for hand hygiene in health-care settings. Recommendations of the MMWR. Recom. Rep. [Internet] 2002; 51(RR-16) [acesso em 08 jan 2016]. Disponível: http://www.cdc.gov/mmwr/pdf/rr/ rr5116.pdf.

11. Mota ÉC, Barbosa DA, de Silveira BRM, Rabelo TA, Silva NM, da Silva PLN, et al. Higienização das mãos: uma avaliação da adesão e da prática dos profissionais de saúde no controle das infecções hospitalares. Rev Epidemiol Control Infect. 2014; 4(1): 12-7.

12. Rosa TP, Magnago TSBS, Lima SBS, Schimidt MD, Silva RM. Perfil dos pacientes atendidos na sala de emergência do pronto socorro de um hospital universitário. R Enferm UFSM. 2011; 1(1): 51-60.

13. do Prado MF, Oliveira ACJ, do Nascimento TMB, de Melo WA, do Prado DB. Estratégia de promoção à higienização das mãos em unidade de terapia intensiva. Ciênc Cuid Saúde. 2012; 11(3): 557-64.

14. dos Santos TCR, Roseira CE, Piai-Morais TH, de Figueiredo RM. Higienização das mãos em ambiente hospitalar: uso de indicadores de conformidade. Rev. Gaúcha Enferm. 2014; 35(1): 70-7.

15. Santos FM, Gonçalves VMS. Lavagem das mãos no controle da infecção hospitalar: um estudo sobre a execução da técnica. Revista Enfermagem Integrada. 2009; 2(1): 152-63.

16. Graf K, Ott E, Wolny M, Tramp N, Vonberg RP, Haverich A, et al. Hand hygiene compliance in transplant and other special patient groups: an observational study. Am J Infect Control. 2013; 41(6): 503-8.

17. Coelho MF, Chaves LDP, Anselmi ML, Hayashida M, Santos CB. Análise dos aspectos organizacionais de um serviço de urgências clínicas: estudo em um hospital geral do município de Ribeirão Preto, SP, Brasil. Rev. LatinoAm Enfermagem. 2010; 18(4): 770-7. 